\title{
Simulation von Förderprozessen bei Vibrationsförderanlagen
}

\author{
Simulation of Conveying Processes in Vibratory Conveyors
}

\author{
Niels Dallinger \\ Thomas Risch \\ Klaus Nendel \\ Professur Fördertechnik \\ Institut für Fördertechnik und Kunststoffe \\ Technische Universität Chemnitz
}

D ie erzielbare Fördergeschwindigkeit bei Vibrationsförderern hängt maßgeblich von der Bewegungsfunktion des Förderorganes ab. Für die gezielte Simulation dieser Anlagen mittels der diskreten Elemente Methode (DEM) ist es notwendig die geometrisch vernetzen Förderorgannachbildungen mit praxisrelevanten Bewegungsfunktionen zu beaufschlagen. Der Artikel beschreibt die Einbindung dieser Bewegungsfunktionen in die quellenoffene DEM-Software LIGGGHTS. Während des Simulationsprozesses wird eine Bewegung vernetzter CAD-Modelle durch trigonometrische Reihen ermöglicht.

[Schlüsselwörter: Vibrationsförderer, Bewegungsfunktion, diskrete Elemente Methode]

$\mathbf{T}$ he conveying velocity achievable with vibratory conveyors depends largely on the conveying pan movement. For the specific simulation of these systems using the discrete element method (DEM), it is necessary to connect the geometric reproductions of conveying pans to practical relevant movement functions. The article describes the integration of movement functions in the open source DEM software LIGGGHTS. A movement of meshed CAD during the simulation process is enabled by trigonometric series.

[Keywords: vibratory conveyor, vibratory movement, discrete element method]

\section{EINLEITUNG}

Vibrationsförderer gehören in das Gebiet der Stetigförderer. Sie werden vor allen Dingen im Bereich der Schüttgüter und Stückgüter mit kleinen bis mittleren $\mathrm{Ab}$ messungen, zur Überwindung kurzer bis mittlerer Strecken eingesetzt. Durch ihre niedrigen Anschaffungs- und Betriebskosten, sowie den geringen energetischen und technischen Aufwand werden sie als häufige Lösung eingesetzt. Weitere Vorteile dieser Technik sind die Möglichkeiten Sortier-, Dosier- und Positionierprozesse in den Förderprozess zu integrieren. Dadurch kann in vielen Fällen auf den Einsatz zusätzlicher Antriebe verzichtet werden.

\section{BEWEGUNGSFORMEN BEI VIBRATIONSFÖRDERERN}

\subsection{D-BEWEGUNGEN VON LINEAREN VIBRATIONSFÖRDERERN}

Ausgehend von der Betrachtung eines linearen Vibrationsförderers (LVF) als Zwei-Massen-Schwingsystem lassen sich die Bewegungen dieser technischen Geräte als zweidimensionale Bewegungsformen darstellen [Ris11].

$\mathrm{Zu}$ jedem Zeitpunkt $t$ kann die Position des Förderorganes durch die zwei Koordinaten $x(t)$ und $z(t)$ abgebildet werden. Abbildung 1 zeigt das vereinfachte Modell eines LVF. Dabei beschreibt $m$ den Massenmittelpunkt. Die zeitabhängigen Bewegungskenngrößen $x(t)$ und $z(t)$ stehen orthogonal zueinander. Durch diese Betrachtung lassen sich die horizontalen und die vertikalen Bewegungen getrennt voneinander bewerten. Mittels der Superposition dieser beiden Bewegungskomponenten wird die Gesamtbewegungsfunktion des Förderorganes als geschlossene Bahnkurve $z(x)$ gebildet. 


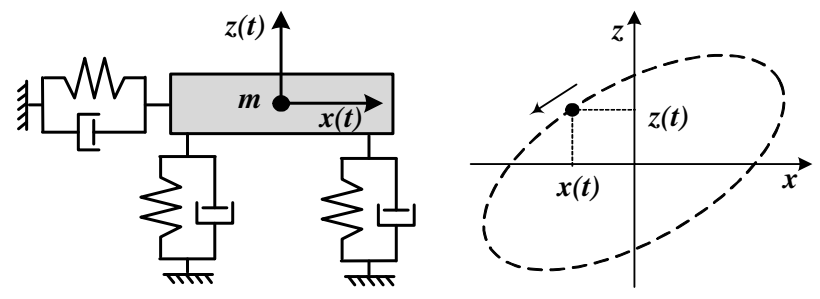

Abbildung 1. Massenpunktmodell eines linearen Vibrationsförderers und die resultierende Bewegungsbahnkurve

Die Bewegung von LVF lässt sich mit diesem Model als Überlagerung zweier harmonischer Sinusschwingungen erster Ordnung beschreiben durch die Gleichungen (1) und (2). Der zeitliche Versatz zwischen dem Erreichen der Amplituden beider Schwingungskomponenten wird als Phasenwinkel $\varphi$ dargestellt.

$$
\begin{gathered}
x(t)=C_{x} \cdot \sin (\omega t) \\
z(t)=C_{z} \cdot \sin (\omega t+\varphi)
\end{gathered}
$$

Einige Vibrationsförderanlagen weisen in der Praxis ausgeprägte Nichtlinearitäten auf. Dies kann durch konstruktive Einflüsse oder durch unharmonische Bewegungsanregungen hervorgerufen werden. Die resultierenden Bewegungsformen solcher Systeme sind in erster Ordnung nicht mehr harmonisch, bleiben jedoch periodisch.

Um die Bewegungsformen erster und höherer Ordnung von LVF beschreiben zu können, werden die periodischen Signale als harmonische Reihen abgebildet. Aus Gründen der besseren Differenzierbarkeit werden mehrgliedrige trigonometrische Reihen in Form von Kosinustermen genutzt. Gleichung (1) kann mittels Gleichung (3) für $n=1$ genauso abgebildet werden wie Gleichung (2) durch (4).

$$
\begin{aligned}
& x(t)=\sum_{n=0}^{k} C_{x n} \cdot \cos \left(n \cdot \omega t+\varphi_{x n}\right) \\
& z(t)=\sum_{n=0}^{k} C_{z n} \cdot \cos \left(n \cdot \omega t+\varphi_{z n}\right)
\end{aligned}
$$

Mittels der Nachbildung von Bewegungsfunktionen als trigonometrische Reihen ist es möglich, nahezu jede erdenkliche Bewegungsform abzubilden. Durch gezielte Approximation der trigonometrischen Bewegungsformen decken diese sich sehr gut mit gemessenen Bewegungskurven bereits bestehender Fördersysteme.

\subsection{BEWEGUNGSFORMEN BEI VIBRATIONSWENDELFÖRDERERN}

Für die Bewegungen von Vibrationswendelförderern (VWF) reicht die vereinfachte zweidimensionale Betrachtung nicht mehr aus. Das Förderorgan der VWF wird durch einen runden Topf gebildet. Dieser besitzt in der
Topfwandung eine oder mehrere Wendeln. Das Fördergut durchläuft während des Betriebes alle drei Raumrichtungen. Die Bewegungsform des Förderorganes entsteht deshalb aus der Superposition der Bewegungskomponenten in $\mathrm{x}-$, $\mathrm{y}$ - und $\mathrm{z}$-Richtung.

Um die Bewegungsform analog zu den LVF darstellen zu können, werden für VWF Zylinderkoordinaten eingeführt. In Abbildung 2 ist ein Vibrationswendelfördertopf schematisch und die Bewegungsform in Abhängigkeit der Zylinderkoordinaten abgebildet.

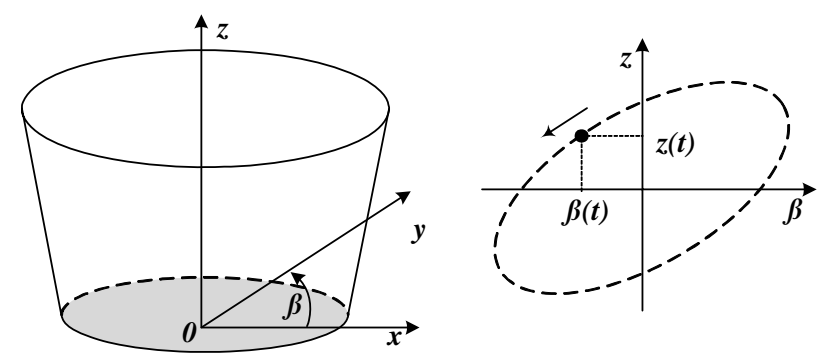

Abbildung 2. Modell eines Vibrationswendelförderers und der dazugehörigen Bewegungsfunktion

Aufgrund der Zylinderkoordinaten lässt sich die Bewegungsform durch einen vertikalen Anteil $z(t)$ in Gleichung (6) und einen Drehschwingungsanteil um die vertikale Achse mit dem Winkel $\beta(t)$ in Gleichung (5) darstellen. In Gleichung (5) stellen die Amplituden $C_{\beta n}$ dementsprechend Winkelausschläge dar.

$$
\begin{aligned}
& \beta(t)=\sum_{n=0}^{k} C_{\beta n} \cdot \cos \left(n \cdot \omega t+\varphi_{\beta n}\right) \\
& z(t)=\sum_{n=0}^{k} C_{z n} \cdot \cos \left(n \cdot \omega t+\varphi_{z n}\right)
\end{aligned}
$$

\section{Diskrete ELEMENTE METHODE}

\subsection{BeDEutung}

Die DEM ist eine numerische Berechnungsmethode, mit deren Hilfe die Bewegungen großer Partikelmengen beschrieben werden können. Sie stammt aus der Notwendigkeit heraus geologische Prozesse mathematisch zu berechnen und wird heutzutage auch für molekulardynamische Simulationen in der Physik verwendet.

Die DEM findet auch im Maschinenbau ein immer breiteres Anwendungsspektrum. Gerade im Gebiet der Fördertechnik und Gutstromanalysen ergeben sich zahlreiche Vorteile. Durch die Berechnung der Newton'schen Gleichungen und Kontakte für jeden einzelnen Partikel, ist es möglich komplexe Gutinteraktionen simulativ zu durchdringen. 


\subsection{GRUNDLAGE DER DEM}

Die DEM beschreibt jeden einzelnen Partikel mittels seiner Masse $m_{p}$, Geschwindigkeit und der auf ihn wirkenden Kräfte. Dabei wird über alle Zeitschritte das Lagransche Kräftegleichgewicht in Gleichung (7) integriert.

$$
\mathrm{m}_{\mathrm{p}} \cdot \ddot{\overrightarrow{\mathrm{x}_{\mathrm{p}}}}=\sum_{\mathrm{i}} \overrightarrow{\mathrm{F}_{\mathrm{i}}}
$$

Die auftretenden Kräfte ergeben sich im makroskopischen Bereich aus Kontaktkräften zwischen den Partikeln untereinander und den Partikel-Wandkontakten. Weitere Kräfte können die Schwerkraft, Reibkräfte sowie Kohäsion und Adhäsion sein.

Für die Beschreibung der gutinternen Partikelkollisionen stehen verschiedene Kontaktmodelle zur Verfügung. Außerdem können jederzeit neue Kontaktmodelle eingebunden werden. Grundlegende Kontaktmodelle sind das Hooke'sche und Hertz'sche Kontaktmodell. Diese Möglichkeiten führen zu einer hohen Flexibilität des nummerischen Simulationsverfahrens.

Die zur Berechnung verwendeten Partikelformen sind in den meisten Fällen idealisierte Kugeln. Komplexere Partikelformen können durch das Verketten von mehreren Kugeln angenähert werden. Dies erhöht die Rechenzeit eines Simulationsmodells deutlich. Außerdem treten durch die idealisierten Annahmen makroskopische Abweichungen zum realen Systemverhalten auf. Dies muss bei der Bewertung der Ergebnisse berücksichtigt werden. Um möglichst realitätsnahe Ergebnisse zu erzielen, müssen die Guteigenschaften experimentell ermittelt und mit idealisierten Gütern (z. B.: Glaskugeln) verglichen werden.

\subsection{HANDHABUNG VON KÖRPERNETZEN}

Für die Simulation von Förderanlagen ist es notwendig, die Geometrie mit Fördergutkontakt in der Simulation abzubilden. In der Software LIGGGHTS werden dafür vernetze CAD-Modelle über eine Schnittstelle integriert. Die zur Verfügung stehende STL-Schnittstelle (surface tesselation language) beschreibt die importierten Oberflächen durch Dreiecke.

Mit den eingebundenen Dreiecksnetzen können verschiedene Kontaktsituationen zwischen Gut und Wand abgebildet werden. Für diese Kontaktpaarungen sind die physikalischen Effekte der Reibung und Scherung in die Software integriert.

Es besteht die Möglichkeit, die Netzgeometrien mit vordefinierten Bewegungen zu beaufschlagen. Diese Bewegungen sind zur Zeit Translation und Rotation sowie translatorische und rotatorische Sinusschwingungen erster Ordnung. Für komplexere Bewegungssituationen besteht die Möglichkeit, variable Bewegungen zu integrieren. In diesem Fall muss der gesamte Berechnungsweg dieser Variablen im Eingabeskript der DEM-Simulation hinterlegt werden.

\section{FUNKTIONSWEISE DER GEOMETRIEBEWEGUNGEN}

\subsection{Einleitung}

Ziel der Arbeit ist es dem Benutzer der Simulationssoftware eine Möglichkeit zu geben, translatorische und rotatorische Schwingungen höherer Ordnung für die Netzbewegung zu verwenden. Für die Integration der trigonometrischen Reihen in die Software LIGGGHTS muss der Quellcode entsprechend erweitert werden. Der Zugriff auf die Softwarequellen ist durch die Verwendung von Öffentlichen Lizenzen (General Public License) möglich. Die Programmiersprache der Softwarequellen ist $\mathrm{C}++$.

Im Folgenden werden die Bewegungen der Netzpunkte im funktionalen Zusammenhang und die Umsetzung der Vektorgeometrie erläutert. Dabei wird aus Gründen der besseren Verständlichkeit nicht näher auf die Umsetzung als $\mathrm{C}++$-Quellcode eingegangen.

\subsection{UMWANDLUNG DER BEWEGUNGSFUNKTIONEN IN VEKTOREN EINZELNER NETZPUNKTE}

Wie bereits in den vorangegangenen Abschnitten erwähnt, handelt es sich bei den in die Simulation importierten CAD-Geometrien um STL-Dateien. Die Dreiecksnetze in den STL-Dateien werden durch die Knotenpunkte der Dreiecke und die dazugehörigen Flächennormalen definiert. Um diese Netze mittels einer softwareinternen Funktion zu bewegen, ist es notwendig, die definierenden Netzknoten zu bewegen.

Die relevanten Bewegungskenngrößen der Netzgeometrien für die Interaktion der Partikel mit den Wänden sind die Positionen der Netzpunkte und deren Geschwindigkeiten in jedem Zeitschritt der Simulation. Diese Kenngrößen müssen durch das Simulationsprogramm berechnet werden.

Die Verschiebung der Netzpunktkoordinaten basiert auf der Vektoralgebra. Im Folgenden wird die Bildung der Orts- und Geschwindigkeitsvektoren für einen Netzpunkt eines beliebigen Wendelfördertopfes beschrieben. Dabei wird von einer wie in Abbildung 2 dargestellten Bewegung ausgegangen. Die Schwingungsbewegungen werden durch die Gleichungen (5) und (6) beschrieben. Dabei wird eine Rotationsschwingung durch eine zur Drehebene orthogonale Translationsschwingung überlagert. Resultierend daraus beschreibt der betrachtete Netzpunkt im dreidimensionalen kartesischen Koordinatensystem eine schraubenförmige Schwingbewegung analog zu Abbildung 3. 

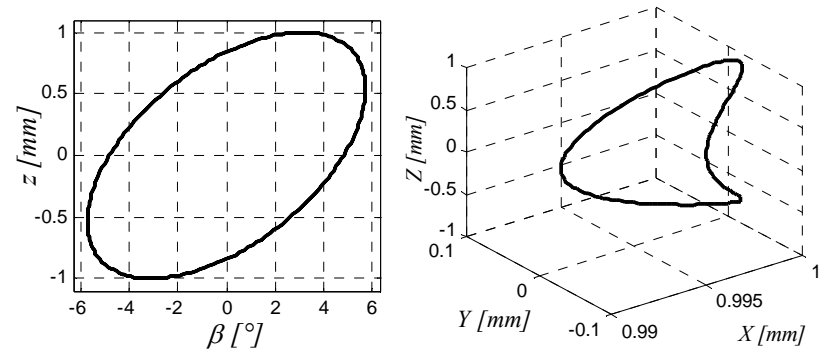

Abbildung 3. Wendelfördererbewegungsfunktion eines beliebigen Netzpunktes mit dazugehörigem Bewegungsverlauf im kartesischen Koordinatensystem

Um die in Abbildung 3 dargestellte dreidimensionale Bewegung zu erzeugen, ist es notwendig, den Netzpunkt in jedem Zeitschritt auf seine neue Position zu verschieben. Abbildung 4 zeigt die notwendigen Vektoren für diese Verschiebung.

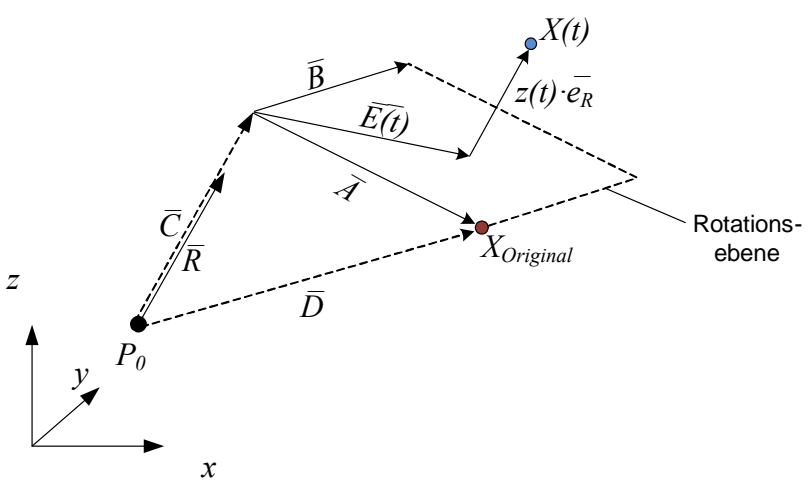

Abbildung 4. Vektoren für die Verschiebung des Originalpunktes entlang der vorgegebenen Bewegung

Für die Rotation und gleichzeitige Translation eines Netzpunktes $X_{\text {Original }}$ ist der Ursprung der Drehachse $P_{0}\left(x_{0}, y_{0}, z_{0}\right)$ und der Vektor der Rotationsachse $\bar{R}$ gegeben. Es handelt sich dabei um die Punktrichtungsform der Drehachse.

Um den vorhandenen Netzpunkt $X_{\text {Original }}$ auf seine neue Position zu verschieben, muss zuerst der Vektor $\bar{D}$ als Strecke zwischen Netzpunkt und Drehachsenursprung gebildet werden.

$$
\overline{\mathrm{D}}=\overline{\mathrm{P}_{0} \mathrm{X}_{\text {Original }}}
$$

Dieser Vektor wird auf die Drehachse projiziert und erzeugt damit den Vektor $\bar{C}$.

$$
\overline{\mathrm{C}}=\left(\overline{\mathrm{D}} \cdot \overline{\mathrm{e}_{\mathrm{R}}}\right) \cdot \overline{\mathrm{e}_{\mathrm{R}}}
$$

Durch die Subtraktion des Vektors $\bar{C}$ von Vektor $\bar{D}$ wird ein senkrecht zur Drehachse stehender Vektor $\bar{A}$ erzeugt.

$$
\bar{A}=\bar{D}-\bar{C}
$$

Um die Ebene der reinen Rotationsschwingung zu definieren, wird ein weiterer orthogonaler Vektor benötigt. Dieser ergibt sich aus dem Kreuzprodukt der normierten Rotationsachse $\overline{e_{R}}$ und dem gerade gebildeten Vektor $\bar{A}$.

$$
\overline{\mathrm{B}}=\overline{\mathrm{A}} \times \overline{\mathrm{e}_{\mathrm{R}}}
$$

Durch diese Vorgehensweise befindet sich der Punkt $X_{\text {Original }}$ immer in der Rotationsebene.

Die Simulationssoftware LIGGGHTS rechnet mit kartesischen und nicht mit Zylinderkoordinaten. Aus diesem Grund wird die Rotation zerlegt. Dabei werden die axialen Anteile in der Rotationsebene laut Gleichung (12) gebildet.

$$
\overline{\mathrm{E}}(\mathrm{t})=\overline{\mathrm{A}} \cdot \cos (\beta(\mathrm{t}))+\overline{\mathrm{B}} \cdot \sin (\beta(\mathrm{t}))
$$

Damit ergibt sich die neue zeitabhängige Koordinate eines Netzpunktes aus Gleichung (13). Der translatorische Schwingungsanteil wird durch den Term $z(t) \cdot \overline{e_{R}}$ beschrieben.

$$
X(t)=P_{0}+\bar{C}+\bar{E}(t)+z(t) \cdot \overline{e_{R}}
$$

Die Geschwindigkeit $\bar{v}(t)$ des Netzpunktes ergibt sich aus der Gleichung (14). Das Kreuzprodukt aus der normierten Drehachse $\overline{e_{R}}$ und der umgewandelten Rotation $\bar{E}(t)$ repräsentiert die Richtung der Tangentialgeschwindigkeit.

$$
\bar{v}(t)=\dot{\beta}(t) \cdot\left(\bar{E}(t) \times \overline{e_{R}}\right)+\dot{z}(t) \cdot \overline{e_{R}}
$$

Alle diese Rechenschritte sind nötig um den Netzpunkt entsprechend $\mathrm{zu}$ verschieben und mit seiner momentanen Geschwindigkeit zu beaufschlagen. Diese Operationen müssen in jedem Zeitschritt der Simulation für jeden Netzknoten durchgeführt werden. Damit lässt sich der hohe Laufzeitanteil der Netzbewegung an der Gesamtsimulation begründen.

\section{ANWENDUNG IN DER SiMULATION}

Die in die Simulationssoftware eingebundenen trigonometrischen Funktionen können für eine Vielzahl von technischen Anwendungsfällen eingesetzt werden bei denen Sinusschwingungen erster Ordnung nicht ausreichen. Gerade aus Sicht der Vibrationsfördertechnik erschließen sich dadurch zahlreiche neue Möglichkeiten der Verwendung von DEM-Simulationen. Es wurde die Möglichkeit geschaffen Simulationsmodelle mit, an die gemessenen Bewegungskurven realer Vibrationsfördereinrichtungen approximierten, Bewegungsformen $\mathrm{zu}$ beaufschlagen und zu simulieren. 
Durch die neuen Bewegungsfunktionen können sowohl Vibrationsförderrinnen als auch Vibrationswendelförderer untersucht werden. Im Bereich von Gutübergabestellen zwischen Vibrationsförderern lassen sich somit im Vorfeld konstruktive Mängel aufdecken. Des Weiteren können speziell zugeschnittene Bewegungen und deren Einfluss auf den Gutstrom untersucht werden. Im Folgenden werden beispielhaft zwei Simulationsmodelle gezeigt.

Abbildung 5 zeigt einen einfachen Vibrationswendelförderer gefüllt mit einem Partikelgemisch. Im Bild sind die Netzstrukturen der Geometrie erkennbar.

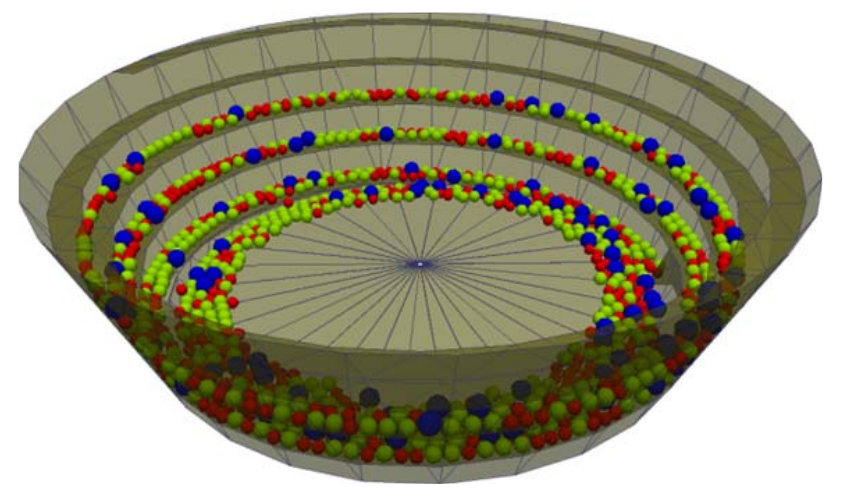

Abbildung 5. Wendelfördertopf mit sichtbarer Vernetzung und Partikelgemisch

Abbildung 6 zeigt eine vereinfachte Wendel als Sortierschiene ausgebildet, welche durch eine Vibrationsrinne gespeist wird. Während der Simulation ordnen sich die verketten Partikel und werden vereinzelt die Wendel aufwärts gefördert. Die Gutabmaße orientieren sich an M6Schrauben.

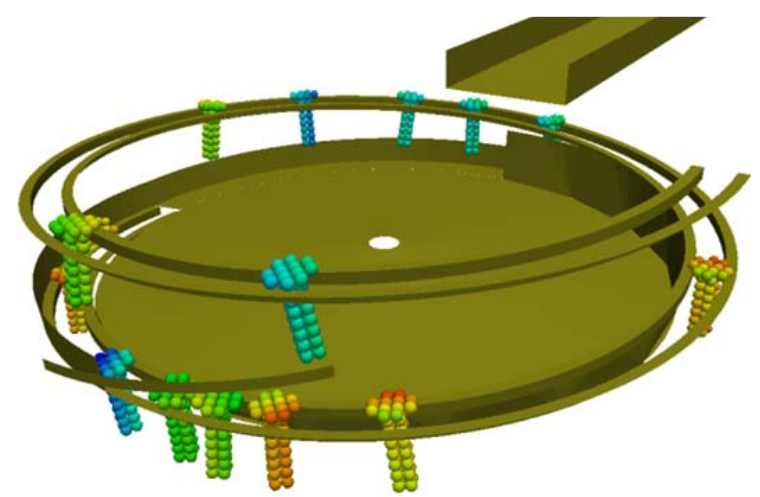

Abbildung 6. Wendelförderschiene mit Ordnungsfunktion am Beispiel von M6-Schrauben
[Loy10]

[Ury08]

\section{LITERATUR}

[Ris11] Risch, Thomas: Zweidimensionale Bewegungsformen in der Vibrationsfördertechnik. Dissertationsschrift, Professur Fördertechnik, TU Chemnitz 2011.

[Bar95] Bartsch, Hans-Jochen: Taschenbuch Mathematischer Formeln. 19.Auflage, Leipzig: Fachbuch Verlag Leipzig, 2001. - ISBN 3-446-21792-4

Loy, Michael: Modulare Vibrationswendelförderer zur flexiblen Teilezuführung. Dissertationsschrift, Lehrstuhl Betriebswissenschaften und Montagetechnik, TU München 2010

Uryadov, Gennady; Katterfeld, André: Simulation der Schüttgutrückwirkung bei Schwingförderern. Tagungsband zum 4. Fachkolloquium der WGTL 2008. -ISBN 978-3-9812554-0-9 